\title{
Research on the Renewal and Development of Public Transport System in Small and Medium-sized Cities of China
}

\author{
Yang Liu \\ Agricultural University of Hebei, HeBei 071000, China. \\ 815959849@qq.com
}

Keywords: Buses, transportation, planning, information, sharing, bus lines, bus rapid transit system.

Abstract: The development level of urban public transport system is one of the factors that affect the urban traffic pressure. In the small and medium-sized cities where the economic superiority is not obvious in China, due to the level of urban infrastructure construction, regional geographical conditions, historical and cultural impact, the city bus is the main choice for how to change and optimize the bus facilities, improve the transport efficiency of public transport and explore and solve other issues.

\section{Introduction}

Urban public transport plays an important role in solving the problem of urban traffic congestion. Because of economic underdevelopment or special historical and geographical conditions, the mainstream of public transport is urban buses. Fixed various points in the city line and each bus station, or bus can take public coin, optimization of city bus, can improve the public convenience of the public transport efficiency, ease road congestion and other pressure, mainly refers to the rail transit, tram, subway, light rail, the latter need more capital operation and supporting infrastructure construction.

In China, cities with less than 100 million of the urban population are small and medium-sized cities, buses are the mainstream, and the construction of public transport cities can ease the pressure of cities and promote the pace of urbanization. At the same time, it is easier to promote construction, and change the efficiency of the situation faster. Therefore, the urban public transit based on the bus system can solve the problem of urban traffic development. Ordinary city bus and station pictures as shown in Figure 1.

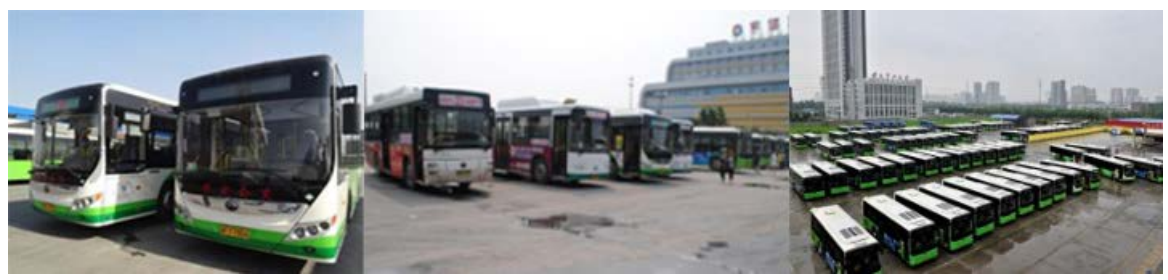

Fig. 1 Ordinary city bus and bus station.

\section{The Problems of Public Transportation in China}

\subsection{Ancillary equipment construction is not complete, update is not timely.}

In the urban renewal stage, the urban infrastructure with the frequency of use of different degrees of destruction and information updates are not timely, for the bus system, the bus stop, bus lines, bus stations and so there will be destroyed, information error, cleanliness Poor, etc., which greatly reduces the bus passenger travel experience, reducing the choice of travel by bus advantage. 


\subsection{The use rates in different periods are different, so there is a low utilization period.}

In the urban office workers, students and the elderly is the main object of the city bus, in the daily peak of the trip, many sites waiting for the number of passengers riding a long time, but a long time to wait for a car, the bus came, passengers Crowded on. This is particularly common at noon working hours every day. And in some places where the bus station is set up, the bus hurries, because the number of passengers on the site is mostly zero, so the meaning of setting up the site does not exist. The distance between the sites also affect the passenger's travel options, how to do a reasonable allocation of the number of passengers to enhance the site, to enhance the experience of riding experience, which is the majority of urban public transport need to consider the issue.

\subsection{Financial investment is inconsistent with the reality of the facilities construction.}

"Bus city" in most of the city's traffic development is applicable, associated with the city's green rise. Government investment in urban public transport system is sufficient to see the importance of the development of public transport, the development of public transport is not only because the public in recent years, the number of public transport routes more and more, the development of public transport, including line adjustment, Adjustment, etc., in addition to these, the policy is also important to point. Financial investment and research data analysis direction should be consistent, rather than blindly increase the number of buses, upgrade the level of public transport equipment.[1]

\section{Advice for the development of innovative public transport}

\subsection{Information Sharing}

Public transport system in the general small and medium cities in the embodiment of passenger cars or gas vehicles as the main means of transport, bus information in the passenger and bus information exchange between the more and more transparent. In the case of the current information network developed, the use of mobile phones, passengers can always understand their choice of bus arrival situation, which is between the bus and passengers is a one-way information exchange, in order to further improve Bus ride efficiency, you can set the bus ride in the site selection system, the use of touch screen, each waiting in the bus waiting for passengers, through the touch to choose their own bus will be riding, so that each will go through this site Of the bus can receive the number of passengers they will accept, as a result of this extension, the bus station information collection can not only be passed to the bus, but also can be used as other information display bus, so that passengers and bus intimacy Increase the number of passengers at the same time the degree of demand for the bus can also be fed back to the bus headquarters, so that can control the bus, share the peak bus pressure.

\subsection{Timely update the sites and lines}

The bus is still a lot of traffic conditions bound or economically underdeveloped cities to strengthen the primary public transport, ordinary bus and bus stations are often immutable, and occasionally road repair and other special circumstances of the temporary bus is often not updated in time, Stop sign is not strong. In the traffic peak and the usual situation, passenger demand and road congestion is different, in crowded urban commercial district and primary and secondary areas, the bus is often in short supply, in the daily three working hours is the same problem.

In order to allow the bus to share more traffic pressure, during the peak demand period, the bus headquarters can increase the number of buses, for the flow of people more sites, you can increase the number of bus lines through this site, the passengers divert. Public transport hub, its location from the qualitative and quantitative point of view of the planning and design, let it play a guiding and guiding role.[2]

\subsection{Bus exclusive lanes}

In most of the bus lanes equipped with bus lanes, bus lanes in the use of the situation, there will be private car occupancy, tampering, grab in front of the bus and so on, and this situation in the policy is ignored, allowed, It is proposed to improve the use of bus lanes of the standard, starting from the traffic management policy, and gradually prohibit the occupation of bus lanes, to enhance the efficiency of bus transport. On the other hand, in the line set, bus lanes and bus stations of the convergence is also more smooth, the bus can stop at any time. In most cities in China are actually 
set up a bus lane, but in the implementation of the situation, to be fully utilized, so as to achieve the purpose of setting. Bus lane in the city set the index can refer to Table 1.

Table 1. Standard for bus lane setting.

\begin{tabular}{ccc}
\hline $\begin{array}{c}\text { Request } \\
\text { classificat } \\
\text { ion }\end{array}$ & Set the index & Specific target requirements \\
\hline $\begin{array}{c}\text { Bus } \\
\text { demand }\end{array}$ & $\begin{array}{c}\text { Bus passenger flow } \\
\text { Road traffic }\end{array}$ & $\begin{array}{c}\text { Peak hour one-way bus traffic } \\
\text { Peak hour bus traffic }\end{array}$ \\
$\begin{array}{c}\text { Road } \\
\text { condition }\end{array}$ & Number of road lanes required & $\begin{array}{c}\text { The proportion of bus passenger flow to road } \\
\text { passenger cars }\end{array}$ \\
$\mathrm{s}$ & & $\begin{array}{c}\text { The number of lanes in a two-way road, the } \\
\text { number of one-way lanes } \\
\text { Road clearance conditions }\end{array}$ \\
\hline
\end{tabular}

\subsection{Planning for fast transit routes}

TOD model refers to the bus priority development model, in the development of small and medium-sized cities, the number of motor vehicles need to control, in the urban planning of traffic planning, for connecting urban commercial pedestrian street, residential center, railway station, passenger station main street, You can plan the bus route, set the bus line at the main point of connection, the line density than other areas to be intensive, to guide the living people, tourists travel options, making the bus line density distribution in the area to meet the operation of the situation, The city commercial center than other regions of the bus line set up, bus distribution more. In order to increase the number of passengers on the bus, set up more suitable for commuting time, according to the site of the reasonable flow of people to narrow or expand the distance between sites, these flexible means easier to operate. [3]

\subsection{Bus facilities and urban aesthetics are closely linked}

Public transport is one of the elements of urban visual surface. The bus station's tidiness, bus station and the cultural factors of urban bus packaging can be optimized from the point of view. Every city has its own cultural atmosphere, affect the city's history, the formation of a commitment to his name card, for example, Baoding, Hebei Province, is a historical and cultural city, but also vigorously promote low-carbon travel, so unified bus system packaging, the bus ride, so tourists. Can play the role of ancient cultural propaganda. Generally speaking, public transportation and related facilities can play the role of city cards, the use of this "mobile billboards", unified colors and text to enhance the city's image.

\subsection{Establishment of a rapid transit system}

The rapid transit system is BRT, and its advance is that it has a high standard of service similar to rail transit and has a flexible operation of conventional ground buses. In the city of rapid transit system should pay attention to the direction of urban development and closely integrated to guide the city in an orderly manner to expand and coordinate the coexistence of ordinary public transport network, both mutually beneficial and win-win situation rather than competitive hostile relations, while the rapid transit system Do not rigidly adhere to a form, we must according to the layout of urban land layout, pay attention to rationality and practicality, according to local conditions, to ensure the feasibility of the line, so that with the direction of urban development closely integrated orderly guide the direction of urban development. For different levels of development of the region, to do the construction standards and specifications, operational efficiency as the first.

\section{Conclusion}

The need for the promotion of public transport in the city Chinese, need to build the backbone of public transport network, and enhance the future development of rail transit connection docking bus better non central area, in response to the state's green travel ideas, provide a positive space to the 
development of city transportation economy, promote transportation planning timely planning, city planning, set up the good image of the city.

\section{References}

[1] Kun Qian, Research on Planning and Design Method of Urban Public Transport Hub,2012.

[2] Tong Li, Guoliang Wang, Yin Jiang, Devoting Major Efforts to Developing Public Transport and Constructing Public Transport System in Central City,2007.

[3] Yongzheng Wang,Jinlong Chu,Land Use Planning Strategy of Urban Residential District under Public Transport, 2009. 